Lastly, in the cerebellum he sees two great functional divisions, a paleocerebellum, which controls the postural or static function of automatic and associated movements, and a neocerebellum for the postural control of isolated synergic movements of cortical origin. [Med. Jl. Australia.]

\title{
2. CRANIAL NERVES.
}

Uhthoff, W. Hemianoptic Disturbances of Visual Field from Gunshot InjurIEs. [Klin. Monatsbl. f. Augenheilkunde, Bd. LV, ro4.]

Four cases of double hemianoptic disturbances of the visual field, of which two were cases of hemianopsia inferior, were observed. Such cases do not occur with thrombotic softening of the brain. The observations led to the assumption that the upper lip of the calcarine fissure corresponded to the upper reticular portion as well as to the lower portions of the visual field. Hemianopsia superior was almost entirely lacking in the material observed. Two patients with homonymous hemianopsia still retained small remnants of the visual field in the blind hemispheres, explicable by the peculiar course taken by the shot. The functional disturbance of the hemianoptically attacked portion of the ocular field varies in intensity, but the expansion of the involved part is always symmetrical. Distinct fatigue phenomena were noted in which the intensity of functional disturbance in the eyc examined first appeared less than in the eye last examined. The half visual field retained was somewhat diminished; concentrically. In all cases except one, the patients had good acuity, although they were temporarily blinded (from $7 / 2$ hours to 4 weeks) by the injury. Recovery in the disturbed field of vision was marked. The ophthalmologic report was normal, provided no intracranial complications arose which might induce the formation of a filling papilla. Total achromasia occurred in only one case. Paralysis of the eye muscles did not occur in any of the nine cases observed. Optic memory was seriously affected in some of the patients, as was also the memory of the period immediately preceding the injury.

Best, F. Hemianopsia and Psychic Blindness in Cranial Injuries. [Gräfes Archiv, I9r7, Bd. 93, No. I.]

Of men semiblinded by the war, 30.2 per cent. had bi-ocular, 25.8 per cent. right-sided, and 44.2 per cent. left-sided hemianopsia. Complete blindness was not found even in bi-ocular semiblindness. Death occurred in 12.8 per cent. of the cases. Monocular semiblindness with straight limit through the focus was met with once, with macula retained, but with complete lapse of the periphery in 3.5 per cent. of the cases; retained periphery and complete lapse of macula in no cases. In recent cases of semiblindness there was preponderating lapse in the lower portion in 58 . I per cent., while in only 5.8 per cent. did the upper preponderate. Paracentral double scotoma without lapse of the periph- 
ery was observed in 3.5 per cent., double scotoma with concomitant peripheral defect in 12.8 per cent., inferiority of the macular region with peripheral lapse in 25.6 per cent., while in 38.4 per cent. only peripheral lapse without paracentral and central disturbance was found. Difficulty in lateral vision was reported in 20.9 per cent. In 18.6 per cent. the pupil on the same side as the lapsed visual field was enlarged, the opposite one in 7 per cent. Nystagmus was present in 10.5 per cent., abducens paralysis (partial) in 4.7 per cent., oculomotor paralysis (partial) in 2.3 per cent., paralysis of all eye muscles in one case. Inaccurate optic localization is the regular accompanying phenomenon of hemianopsia, the simplest test for this, halving of distances, was carried out typically in 24. .I per cent. (the shortest distance on the side of the semiblindness), and atypically in 36 per cent. In 31.3 per cent. of the cases the semiblindness was associated with a disturbance of optic comprehension (optic numerical disturbance), in 5.8 per cent. with optic agnosia. In 25.6 per cent. alexia accompanied the disturbances, and agraphia in 14 per cent.

When an incomplete semiblindness occurs, as is usually the case in injury of the calcarine region, all visual functions in that sector, as well as all perception of bright colors and light, together with peripheral acuity, is disturbed. There is no particular center for bright colors in so far as it concerns agnostic, amnestic and aphasic disturbances of the color sense. The seat of the cerebral center of vision for optic spatial perception is the calcarine. The location of the cerebral macula could not be determined. Replacement on each half of the macula in both cerebral hemispheres is however highly improbable. The rarity of total lapse of half the visual field is explained by the relatively great extent of the calcarine, the lower portion of which is almost never wholly destroyed, while the patient lives, on account of the proximity of the cerebellum. This explanation also serves for the rarity of complete failure of macular stimuli.

Optic reflex eye motions, especially functional motions and convergence, are produced from the calcarine. This is the center of relative optic localization, in brain injuries to this portion relative and absolute disturbances of localization arise, an analysis of which the author has attempted. Other cortex regions must be taken into account together with the calcarine, for the localization of visual objects. Almost all spatial relations in reference to the body are lost without these. Other visual disturbances, alexia and agraphia, are connected with left-sided brain injury. They have no common memory field however. Primitive optic-agnostic disturbances (including numerical disturbances) are observed in injuries of both the right and left brain. Acute psychic blindness only occurred in injury to both sides. Spatial disturbances of optic recognition are connected with injuries to cortical fields other than those of the amnestic-agnostic disturbances. 